\title{
Self-excitation analyses of vocal chord under breathing flow and frequency change by muscle activation
}

\author{
T. Tsuta ${ }^{1}$, T. Iwamoto ${ }^{1}$, T. Shimizu ${ }^{2} \&$ D. Egusa ${ }^{2}$ \\ ${ }^{I}$ Department of Clinical Engineering, Faculty of Health Science, \\ Hiroshima International University, Japan \\ ${ }^{2}$ Graduate School of Mechanical Engineering System, \\ Hiroshima University, Japan
}

\begin{abstract}
This paper presents some analyses and experiments on vocalizing mechanics in the vocal chord due to the breathing flow, and their wide range of frequency change by varying the interior muscle activations.

Three-dimensional dynamic responses of the hyperelastic and finite deformation body of the vocal chord and the interior muscles have been analysed at first and the exclusive FEM system has been developed. The eigen frequencies and vibration characteristics of the vocal chord are analysed by varying the activation level of the interior muscles.

The Helmholtz equation of the breathing flow under transient state has been analysed to obtain the pressure pulsation characteristics behind the vocal chord during vocalizing of the source voice. Combining both approaches stated above, the mechanism of the self-exciting vibration of the vocal chord has been studied in detail using the route-locus approach.

It is also found in the analysis and experiments that the frequency of the selfexciting vibration can be widely varied with the activation level of the interior muscle of the vocal chord, giving rise to a variety of frequency levels of the source voice.
\end{abstract}

\section{Introduction}

It is important to clarify theoretically the human's vocalizing mechanics in the larynx-vocal tract system, in order to develop the artificial system for a 
disordered person, automatic translation system and so on. Past approaches mainly concerns with respect to the signal processing after pronunciation, and it is not easy to apply them due to personal pronunciations with the enormous types of different pronunciation patterns. However once such vocalizing mechanics of living human has been clarified in theoretically and applied to advanced artificial larynx system in successfully, their contribution to people with disordered pronunciation, could be remarkable in the field of assistive technology in medical engineering.

Theoretical approach on vocalizing mechanics in vocal chord had been done not so many in comparison with experimental works for living human. IshizukaFlanagan solved the problem using the model with 2DOF and two mass systems, orthogonal to the breathing flow. Kagawa et al solved the vocal chord vibration using the 2D finite element approach. However it does not include the influences of vocal muscle activation and relative motion of some cartilage. Since the pressure pulsation of breathing flow and eigenfrequency characteristics of vocal chord are coupled to generate the self-excitation vibration characteristics of vocal chord, and that the self-exciting frequency vary with the stiffness change in vocal chord, which is activated by the interior and/or exterior muscle activations.

In this paper, three-dimensional finite element approach for analysing eigenfrequency and dynamic response of muscle and hyperelastic finite-deformation soft tissue have been developed at first (Figure 1). Combined it with the Lighthill equation of 3D potential-flow of breathing flow from lung to the vocal-chord, a total system to solve the self-exciting vibration of vocal chord has been developed, and it is applied to solve wide varieties of the frequency of selfexciting vibration of the vocal-chord, which vary with the activation of the interior muscles of the vocal-chord. It is demonstrated, through comparisons with calculation and measurement results of the vocal-chord vibrations and the pulsating flow, at the mouth outlet part in vocalizing of a living human, 22 years old, that the simulation results, obtained by the above method, can be satisfactorily applied to solve this kind of problems.

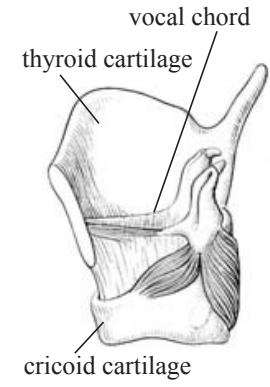

(a) a view of larynx

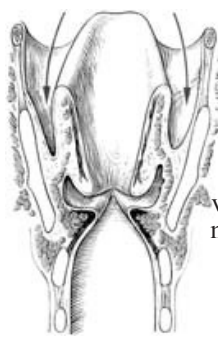

(b) sectional view

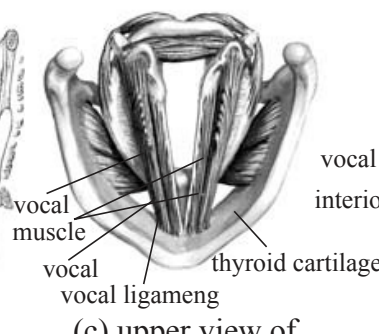

(c) upper view of vocal muscle

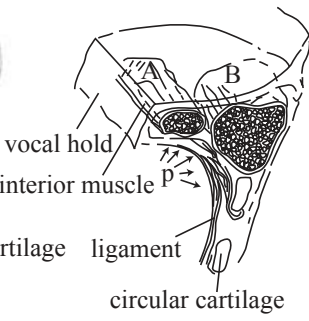

(d)

Figure 1: Vocal chord and interior muscles. 


\section{Analysis of muscle-ligament system}

\subsection{Active and passive constitutive modeling of muscle}

Based on the Hill type 3 element system (Figure 2(a)), the vocal muscle element(C.E.) contract actively due to the neuro stimulation $\beta_{2}$ with the amount $l_{0}\left(\beta_{2}\right)$ under the isotropic contraction as in Figure 2(e). However, when the external force $f^{e}$ is loaded to the system, a part of the free contraction, is constrained with the amount of $\Delta l\left(\beta_{2}\right)$, so as to generate the counter force $f^{C E}\left(\beta_{2}\right)$, and the free contraction reduces from $l_{0}\left(\beta_{2}\right)$ to $l\left(\beta_{2}\right)$ That is, the free contraction $l_{0}\left(\beta_{2}\right)$ is shared partially for generating the force $f^{C E}\left(\beta_{2}\right)$ and the other for generating the required motion $l\left(\beta_{2}\right)$ in force-motion and activation space.

By neglecting the series element (S.E.) in Figure 2(a) for simplicity, the active and passive constitutive law of muscle has been formulated, based on the Hatze's constitutive law for single muscle cell, shown in equations (1) and (2), for the mth muscle shown in Figure 2(b). Based on Green strain $E_{i j}$ and Kirchhoff stress $S_{i j}$, the multi-axial active constitutive model can be derived from the evolutional model of Hatze(1) and can be written by equations (3) and (4).

$$
\begin{gathered}
F^{C E}=F_{0} B(\beta) F(E) G(v) \\
B(\beta)=a_{k} \exp \left[b_{k} B(\beta, E)\right](k=1,2) \\
G(v)=\left(G_{0}+a\right) b /(v+b)-a \\
F(E)=c+\bar{c} \exp [R(E)] \sin [V(E)] \\
R(E)=-\mu_{1} E, V(E)=\mu_{2}\left(E+c_{3}\right) \\
S_{i j}^{a m}=D_{m}^{a}\left(\beta, E, E_{0}, \dot{E}\right)\left\{E_{i j}-\delta_{m j} E_{o i}\right\} \\
\left.\left.D_{m}^{a}\left(\beta, E, E_{0}, \dot{E}\right)=S_{0} B(\beta) F\left(E, E_{0}\right) G\right) \dot{E}, \dot{E}_{0}\right)
\end{gathered}
$$

where $F_{o}$ is the maximum contraction force under isometric condition, and $B(\beta), F(E)$ and $G(V)$ are functions expressing activation rate, contraction force $\sim$ deformation, and force-velocity relations, respectively. The functions $B(\beta)$ and $F(E)$ are schematically expressed as in Figure 3(d) and 3(e), respectively, and $G(V)$ is given by Hill [4]. The other notations, $a_{k}, b_{k}$, are experimental constants. The suffix $m$ means $m$-th muscle shown in Figure 2(b), and $E_{0 i}$ is the free contraction strain equivalent to $l_{0}\left(\beta_{2}\right)$ in Figure 2(e).

In the next place, the passive stress $S_{i j}^{p}$ can be determined from strain energy function $W\left(J_{i}\right)_{m}=1 / 2 C_{m} \exp \left[Q^{P}\left(E_{i j}\right)\right]$ as in Equation (5) where $J_{i}$ is the i-th invariant of the strain tensor and $Q^{P}=E_{i j}^{T} D_{m} E_{i j}$ [5].

$$
\begin{aligned}
S_{i j}^{p m} & =\frac{\partial W_{m}}{\partial E_{i j}}=\frac{\partial W_{m}}{\partial J_{k}} \frac{\partial J_{k}}{\partial E_{i j}} \\
& =2 C_{m}\left[D_{m}^{p}\right] \exp \left[Q^{P}\left(E_{i j}\right)\right] \cdot\left\{E_{i j}\right\}=\left[D_{i m}^{P}\right]\left\{E_{i j}\right\}
\end{aligned}
$$


where $C_{m}$ is the material constant. The combined stress $S_{i j}^{m}$ can be obtained from equations. (3) and (5) as in equation (6), i.e.

$$
\begin{gathered}
\left\{S_{i j}^{a m}\right\}+\left\{S_{i j}^{p m}\right\}=\left[D_{m}^{a}\right]\left\{E_{i j}-\delta_{m j} E_{o j}\right\}+\left[D_{m}^{p}\right]\left\{E_{i j}\right\}+h\left\{\frac{\partial I_{3}}{\partial E_{i j}}\right\} \\
{\left[S_{i j}^{L}\right]=[L]^{T}\left[S_{i j}^{m}\right][L] \quad \text { or } \quad\left\{S_{i j}^{L}\right\}=[T]\left\{S_{i j}^{m}\right\}}
\end{gathered}
$$

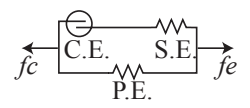

(a) Hill's 3-el. model

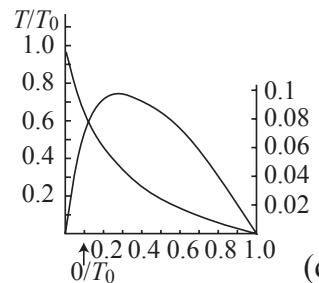

(c) velocity force curve

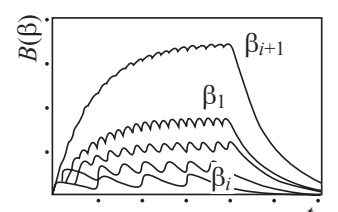

(d) activation dynamics $\stackrel{t}{\text { curves }}$
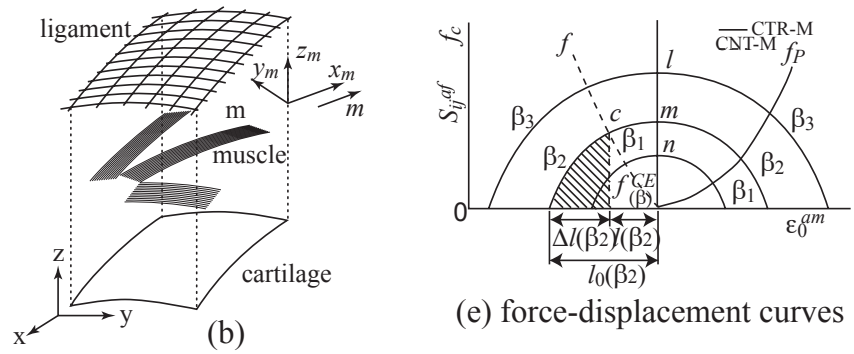

(e) force-displacement curves

Figure 2: The stimulation and contraction deformation characteristics of the muscle.

where $I_{3}$ is the 3rd-invariant of the deformation tensor and expressed by $I_{3}=1+2 J_{1}+4 J_{2}+8 J_{3}$ and $h$ is the Langrange parameter for expressing the incompressibility of the muscle. The stress in equation (6), expressed in the muscle coordinate shown in Figure 2(b), can be rewritten in the local coordinate system using coordinate transformation matrices [L] or [T], as in equation (7)' or (7).

\subsection{Incremental stiffness equation for muscle-ligament system}

The incremental form of stress and strain in muscle can be written by neglecting the notation $\mathrm{L}$ as

$$
S_{i j}=S_{i j}^{n-1}+\Delta S_{i j}^{n}, \text { and } E_{i j}=E_{i j}^{n-1}+\Delta E_{i j}^{n},
$$

where, neglecting to denote $n$, 


$$
\begin{aligned}
& \Delta E_{i j}=\frac{1}{2}\left\{\frac{\partial \Delta u_{i}}{\partial X_{j}}+\frac{\partial \Delta u_{i}}{\partial X_{i}}+\frac{\partial u_{k} \partial \Delta u_{k}}{\partial X_{i} \partial X_{j}}+\frac{\partial \Delta u_{k}}{\partial X_{i}} \frac{\partial u_{k}}{\partial X_{j}}\right\} \\
&\left\{\Delta S_{i j}\right\}=\left(\left[\frac{\partial D_{m}^{a}}{\partial \beta}\right] \Delta \beta^{n}+\left[\frac{\partial D_{m}^{a}}{\partial E_{i j}}\right] \Delta E_{i j}^{n}+\frac{\partial D_{m}}{\partial \dot{E}_{i j}} \Delta \dot{E}_{i j}\right)\left\{\left(E_{i j}^{n-1}+\Delta E_{i j}^{n}\right)\right. \\
&\left.-\left(\delta_{m i} E_{0 m}^{n-1}+\delta_{m i} \Delta E_{0 m}^{n}\right)\right\}+\left[D_{m}^{a}\right]\left\{\Delta E_{i j}^{n}-\delta_{m i} \Delta E_{0 m}^{n}\right\} \\
&+\left[\frac{\partial D^{p}}{\partial E_{i j}}\right] \Delta E_{i j}^{n}\left(\left\{E_{i j}\right\}^{n-1}+\left\{\Delta E_{i j}^{n}\right\}\right)+\left[D^{p}\right]\left\{\Delta E_{i j}^{n}\right\} \\
&+\frac{\partial I_{3}}{\partial E_{i j}}\{\Delta h\}
\end{aligned}
$$

where $u_{i}$ is the displacement, $X_{i}$ the coordinate before deformation.

Then, the incremental virtual work equation can be written by neglecting the notation $n$, as

$$
\begin{gathered}
\int_{v}\left\{\delta \Delta E_{i j}\right\}^{T}\left(\left\{S_{i j}^{a}+\Delta S_{i j}^{a}\right\}+\left\{S_{i j}^{p}+\Delta S_{i j}^{p}\right\}\right) d v+\int_{v}\{\delta \Delta h\}\left\{I_{3}+\Delta I_{3}\right\} d v \\
=\int_{v}\left\{\delta \Delta u_{i}\right\}^{T}\left\{T_{0 i}+\Delta T_{0 i}\right\} d s+\int_{v}\left\{\delta \Delta u_{i}\right\}^{T}\left\{F_{0 i}+\Delta F_{0 i}\right\} d v
\end{gathered}
$$

where $T_{0 o}$ and $F_{0 i}$ are body force and surface force.

Using equations (10) and (7), the incremental stiffness equation of an element can be rewritten in the form

$$
\left[\hat{K}^{L}\right]\left\{\begin{array}{l}
\Delta \hat{u} \\
\Delta h
\end{array}\right\}=\left\{\Delta p_{a}\right\}+\{\Delta p\}+\{R\}=\{\hat{\Delta} p\}
$$

where

$$
\begin{aligned}
& {\left[\hat{K}^{L}\right]=\int_{v}\left[\begin{array}{ccc}
\hat{B}^{T} & B^{T} & 0 \\
& 0 & 1
\end{array}\right]\left[\begin{array}{cccc}
T^{-1} & \Delta D_{T} T & T & D_{e v} \\
& D_{e v} & & 1
\end{array}\right]\left[\begin{array}{cc}
B \hat{B} & 0 \\
0 & I
\end{array}\right] d v}
\end{aligned}
$$

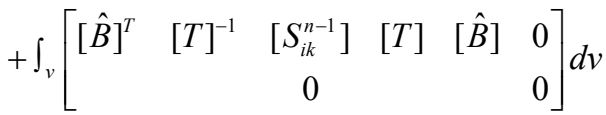

$$
\begin{aligned}
& {\left[S_{i k}^{n-1}\right]=\left[S_{i k}^{a n-1}\right]+\left[S_{i k}^{p n-1}\right] \text {, }} \\
& \left\{\Delta p_{a}\right\}=\int_{v}\left[\hat{B}^{T} B^{T}\right][T]^{-1}\left[\Delta D_{T}^{a}\right][T]\left\{\Delta E_{0 m}^{n}\right\} d v \\
& \Delta D^{T}=\Delta D_{T}^{a}+\Delta D_{T}^{p}, \\
& \{\Delta p\}=\int_{s}[N]^{T}\left\{\Delta T_{0 i}\right\} d s+\int_{v}[N]^{T}\left\{\Delta F_{0 i}\right\} d v
\end{aligned}
$$

and $R$ is the residual force up to the step, $n-1, N$ the shape function, $[B]$ and $[\hat{B}]$ being given in $[2]$. The other notations are given by 


$$
\begin{aligned}
& \Delta D_{T}^{a}=\sum_{i=1}^{4} \Delta D_{i}^{a}, \quad \Delta D_{T}^{p}=\sum_{i=1}^{2} \Delta D_{i}^{p}, \Delta D_{1}^{a}=\frac{\partial D_{m}^{a}}{\partial \beta} \Delta \beta, \\
& \Delta D_{2}^{a}=\frac{\partial D_{m}^{a}}{\partial E_{i j}} \Delta E_{i j}, \Delta D_{3}^{a}=\frac{\partial D_{m}^{a}}{\partial \dot{E}_{i j}} \Delta \dot{E}_{i j}, \Delta D_{4}^{a}=D_{k}^{a}, \\
& \Delta D_{1}^{p}=\frac{\partial D_{m}^{p}}{\partial E_{i j}} \Delta E_{i j}, \Delta D_{2}^{p}=D_{i j}^{p}, \quad D_{e v}=\frac{\partial I_{3}}{\partial E_{i j}}
\end{aligned}
$$

By assembling element stiffness equation (12) for whole elements, and transforming them by global coordinate system, we obtain the governing stiffness equation of whole muscles.

It should be noted that in equation (12), the load vector $\left\{\Delta P^{a}\right\}$ is the force induced by the pull back of muscle free contraction, and it is especially important to obtain the exact stress in muscle.

\section{Dynamic equation of motion for vocal soft, tissue}

\subsection{Constitutive equation of vocal soft, tissue}

The coordinate system is defined as shown in Figure 3, and $x^{-} y$ plane is defined to be the spreading direction and $\mathrm{z}$ to be the thickness direction.

The Green strain $E_{i j}$ and kirchhoff stress $S_{i j}$ can be expressed by

$$
\begin{gathered}
E_{i j}=\frac{1}{2}\left(\frac{\partial u_{i}}{\partial X_{j}}+\frac{\partial u_{j}}{\partial X_{i}}+\frac{\partial u_{m}}{\partial X_{i}} \frac{\partial u_{m}}{\partial X_{j}}\right) \\
\left\{E_{i j}\right\}=[\tilde{B}]\{\bar{u}\}
\end{gathered}
$$
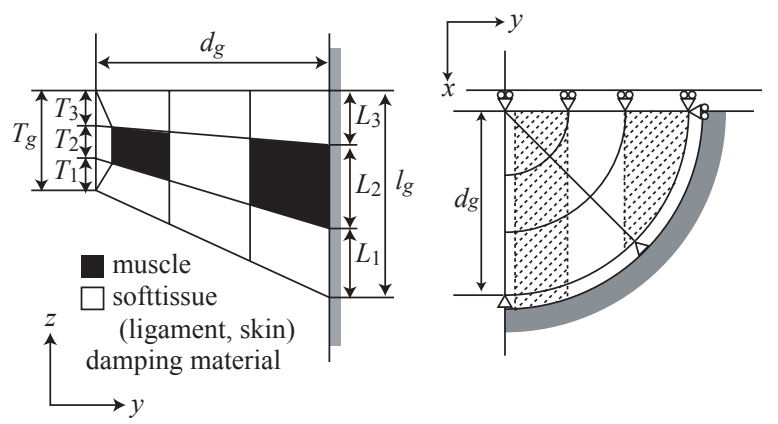

\begin{tabular}{|lll|}
\hline radeius & $d_{g}$ & 9.000 \\
\hline thickness & $T_{g}$ & 3.000 \\
& $T_{1}$ & 1.000 \\
& $T_{2}$ & 1.000 \\
& $T_{3}$ & 1.000 \\
\hline length & $L_{g}$ & 8.000 \\
& $L_{1}$ & 3.000 \\
& $L_{2}$ & 3.000 \\
& $L_{3}$ & 2.000 \\
\hline
\end{tabular}

Figure 3: Calculation model of vocal chord.

$$
S_{i j}=\left(\frac{\rho_{o}}{\rho}\right)\left(\frac{\partial X_{i}}{\partial x_{m}}\right)\left(\frac{\partial X_{j}}{\partial x_{n}}\right) \sigma_{m n}
$$


where $u_{i}(\mathrm{i}=1,2,3$ which is replaced to be $\mathrm{x}, \mathrm{y}, \mathrm{z})$ is the displacement, $X_{i}$ and $x_{i}$ the coordinate system before and after deformation, $\rho_{o}$ and $\rho$ the densities and $\sigma_{m n}$ the true stress respectively.

Then the constitutive equation can be written by

$$
\begin{aligned}
\left\{S_{i j}\right\} & =\left[D_{i j}{ }^{k l}\right] \cdot\left\{E_{i j}\right\} \\
& =\left(D_{A}+c D_{B} \exp \left[G\left(\mu_{m}, E_{i j}\right)\right]\right) \cdot\left\{E_{i j}\right\}
\end{aligned}
$$

where

$$
\begin{aligned}
G\left(\mu_{m}, E_{i j}\right)=\mu_{a} E_{11}{ }^{2}+\mu_{b} E_{22}{ }^{2}+\mu_{c} E_{33}{ }^{2} \\
+\mu_{d} E_{12}{ }^{2}+\mu_{e} E_{23}{ }^{2}+\mu_{f} E_{31}{ }^{2} \\
+2 \mu_{g} E_{11} E_{22}+2 \mu_{h} E_{22} E_{33}+2 \mu_{i} E_{33} E_{11} \\
D_{A}=\left[\begin{array}{cccccc}
\alpha_{a} & \alpha_{g} & \alpha_{i} & 0 & 0 & 0 \\
\alpha_{g} & \alpha_{b} & \alpha_{h} & 0 & 0 & 0 \\
\alpha_{i} & \alpha_{h} & \alpha_{c} & 0 & 0 & 0 \\
0 & 0 & 0 & \alpha_{d} & 0 & 0 \\
0 & 0 & 0 & 0 & \alpha_{e} & 0 \\
0 & 0 & 0 & 0 & 0 & \alpha_{f}
\end{array}\right] \\
D_{B}=\left[\begin{array}{cccccc}
\mu_{a} & \mu_{g} & \mu_{i} & 0 & 0 & 0 \\
\mu_{g} & \mu_{b} & \mu_{h} & 0 & 0 & 0 \\
\mu_{i} & \mu_{h} & \mu_{c} & 0 & 0 & 0 \\
0 & 0 & 0 & \mu_{d} & 0 & 0 \\
0 & 0 & 0 & 0 & \mu_{e} & 0 \\
0 & 0 & 0 & 0 & 0 & \mu_{f}
\end{array}\right]
\end{aligned}
$$

In the above, $\alpha, \mathrm{M}$ and $\mathrm{C}$ are material constants.

\subsection{Incremental form of the equation}

The green strain on the n-th step $\Delta E_{i j}{ }^{n}$ can be expressed by

$$
\Delta E_{i j}{ }^{n}=\frac{1}{2}\left\{\frac{\partial \Delta u_{i}}{\partial X_{j}}+\frac{\partial \Delta u_{j}}{\partial X_{i}}+\frac{\partial \Delta u_{k}^{n-1}}{\partial X_{i}} \cdot \frac{\partial \Delta u_{k}^{n}}{\partial X_{j}}+\frac{\partial \Delta u_{k}^{n}}{\partial X_{i}} \cdot \frac{\partial \Delta u_{k}^{n-1}}{\partial X_{j}}\right.
$$

or in matrix form as

$$
\left\{\Delta E_{i j}\right\}=[B]\left(\frac{\partial \Delta u_{j}}{\Delta X_{k}}\right)=[B][\hat{B}]\{\Delta \bar{u}\}
$$

where $\bar{u}_{i}$ is the nodal displacement, $\mathrm{N}$ the shape function of the FEM, and $\hat{B}, B$ the strain-nodal displacement matrices, respectively. 
The incremental form of the constitutive model is given by

$$
\left\{\Delta S_{i j}{ }^{n}\right\}=\left[D_{h e}{ }^{n-1}\right]\left\{\Delta E_{i j}{ }^{n}\right\}
$$

where

$$
\begin{aligned}
{\left[D_{h e}{ }^{n-1}\right]=} & D_{A}+c \cdot D_{B} \cdot \exp \left[G\left(\mu_{m}, E_{i j}\right)\right]_{n-1} \\
& +c . D_{B}\left\{E_{k l}{ }^{n-1}\right\}\left\{\frac{\partial \exp \left[G\left(\mu_{m}, E_{i j}\right)\right]_{n-1}}{\partial E_{k l}}\right\}^{T}
\end{aligned}
$$

\subsection{Discretized dynamic equation of motion}

Dynamic virtual work equation of motion of vocal chord can be expressed by

$$
\int_{v} \delta u_{i} \rho_{o} \ddot{u}_{i} d v+\int_{v} S_{i j \delta E_{i j}}=\int_{s} T^{0 i} \delta u_{i} d s+\int_{v} F^{0 i} \delta u_{i} d v
$$

where $T_{i s}^{o}$ the surface traction, $F^{o}$ the body force, and $\delta$ the virtual value, respectively.

From equation (15), we have the equation in matrix form where $[\widetilde{B}]$ is the Green strain-nodal displacement matrix.

Then the equation (23) can be rewritten in matrix form as

$$
[\bar{M}]\{\dot{\Delta} \dot{\bar{u}}\}+[\bar{C}]\{\dot{\Delta} \bar{u}\}+[\bar{K}]\{\Delta \bar{u}\}=\{\Delta \bar{P}\}
$$

where

$$
\begin{aligned}
& {[\bar{M}]=\sum \int_{v}[N]^{T} \rho_{0}[\hat{N}] d v_{e},[\bar{K}]=\sum \int_{v}[\tilde{B}]^{T}\left[D_{i j}{ }^{k l}\right][\tilde{B}] d v_{e}} \\
& {[\bar{C}]=\eta[K], \quad\{\bar{P}\}=\sum \int_{s}[N]^{T}[N]\{\bar{p}\} d s=[G]\{\bar{p}\}}
\end{aligned}
$$

\section{Lighthill equation for pulsating flow behind the vocal chord}

Combined with the conservative laws of mass and momentum, the Lighthill equation for the pulsating flow from the lung for vocalizing can be written by

$$
\nabla^{2} p_{a}-\frac{1}{c_{o}^{2}} \frac{\partial^{2} p_{a}}{\partial t^{2}}=\frac{\partial^{2} T_{i j}}{\partial x_{i} \partial x_{j}}
$$

where

$$
T_{i j}=p u_{i} u_{j}, p-p_{o}=c_{o}^{2}\left(p-p_{o}\right)
$$

The right hand side of equation (26) is the sound source term, and $T_{i j}$ is the Lighthill's stress tensor for turbulent flow. The suffix "o" denotes the steady flow quantities, and $\mathrm{pa}=\mathrm{p}-p_{o}$ is the pulsating pressure component, $c_{o}$ the sound velocity and $u_{i}$ the flow velocity respectively. The boundary conditions of flow at the boundary $\Gamma_{f a}$ shown in Figure 4 can be expressed by

$$
\frac{\partial p_{a}}{\partial n}=\rho_{a} \ddot{u}_{a} \text { on } \Gamma_{f a}, \frac{\partial p_{a}}{\partial n}=0 \text { on } \Gamma_{n}
$$


where $n$ is the outward normal vector of the boundary. Multiplying the weighting function $\Phi$ to equations (26) and (27), the weighted residual equation in weak form can be expressed by

$$
\int_{V_{a}} \phi\left(\nabla^{2} p_{a}-\frac{1}{c_{a}^{2}} \ddot{p}_{a}-Q\right) d v=\int_{\Gamma_{f a}} \phi\left(\frac{\partial p_{a}}{\partial n}-p_{a} \ddot{u}_{a}\right) d S
$$

where $Q=\partial^{2} T_{i j} / \partial u_{i} \partial u_{j}$ is the sound source term and is assumed to be invariant. Using the shape functions to be

$$
p_{a}=\sum_{j} N_{a j} p_{a j}, Q=\sum_{j} N_{a j} Q_{j}, \phi=\sum_{i} N_{a i} p_{a i}
$$

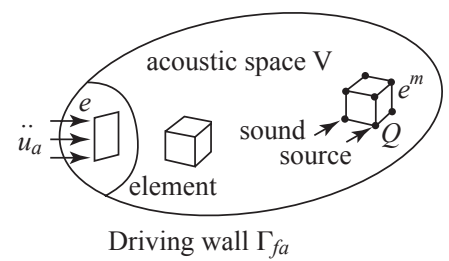

Figure 4: Acoustical space model.

and based on the Gauss divergence theorem, the equation (28) can be written in discretized form as

$$
\begin{gathered}
\sum_{e}\left(\left\{p_{a}\right\}_{e}^{T}[H]_{e}\left\{p_{a}\right\}_{e}+\left\{p_{a}\right\}_{e}^{T}[G]_{e}\left\{\ddot{p}_{a}\right\}_{e}\right) \\
=\sum_{e}\left(\left\{p_{a}\right\}_{e}^{T}\left\{F_{a}\right\}_{e^{\prime}}\right)
\end{gathered}
$$

where

and

$$
\begin{gathered}
H_{a i j}=\int_{e}\left(\frac{\partial N_{a i}}{\partial x} \frac{\partial N_{a j}}{\partial x}+\frac{\partial N_{a i}}{\partial y} \frac{\partial N_{a j}}{\partial y}+\frac{\partial N_{a i}}{\partial z} \frac{\partial N_{a j}}{\partial z}\right) d V \\
G_{a i j}=\frac{1}{c_{a}^{2}} \int_{e} N_{a i} N_{a j} d V
\end{gathered}
$$

from which the governing equation for whole system can be written as follows by adding the damping term to be proportional to [Ha] matrix (where $\alpha$ the proportional constant),

$$
[\mathrm{Ga}]\{[G a]\{\ddot{p} a\}+\alpha[H a]\{p a\}+[H a]\{p a\}=\{F a\}
$$


In case of the transient problem, the equation (31) is further discretized with respect to time, we can solve it incrementally, using the Newmark- $\beta$ method. In case of steady problem, substituting the expression $p_{a}=p_{\widetilde{a}} e^{j \omega t}$ into equation (31), we can solve it for the amplitude vector $\{p \widetilde{a}\}$ as

$$
\left[\begin{array}{cc}
{[H a]-\omega^{2}[G a]} & -\omega \alpha[H a] \\
\omega \alpha[H a] & {[H a]-\omega^{2}[G a]}
\end{array}\right]\left\{\begin{array}{l}
p a_{R} \\
p \tilde{a}_{J}
\end{array}\right\}=\left\{\begin{array}{l}
{[F a]_{R}} \\
{[F \tilde{a}]_{J}}
\end{array}\right\}
$$

where suffixes $R$ and $J$ means real and imaginary terms respectively.

\section{Self excitation vocalization analysis}

During a cycle, a vocal chord under closed state is subjected to the pressure rise due to the breathing flow from the lung and when it reaches to a critical level, the lip open and the pressure is released, yielding to the next lip-closure. The selfexciting vibration is occurred if this cycle of iteration coincides with the eigenfrequencies of the vocal chord and that it is driven under steady amplitude vibration condition.

Three dimensional finite element system for solving dynamic response of arbitrary hyperelastic and finite deformation solid applicable to vocal chord has been developed at first.

The computer system to solve the Helmholtz equation has been developed in order to calculate the pressure pulsation in breathing flow behind the vocal chord.

Based on the above, the combined system for solving coupled dynamic response of vocal chord and for solving pulsating pressure in breathing flow has been developed.

The configuration of a vocal chord of a living human, 22 years old, including the surrounded ligaments and the interior and exterior muscles is obtained by MRI measurement and discretized into finite elements.

A static stress analysis of the vocal chord has been done at first to get the stiffness of the vocal chord under interior muscle activation, and a series of eigen frequency analysis has been carried out under various muscle activation.

The governing dynamic equations of motions for both the vocal chord and the breathing flow are solved in coupled form as follows

$$
\begin{aligned}
& \left.\begin{array}{l}
M \dot{\bar{v}}+C \bar{v}+K \bar{u}=G \Delta \bar{p} \\
\bar{v}-\dot{\bar{u}}=0
\end{array}\right\} \text { for vocal chord } \\
& \left.\begin{array}{l}
M_{a} \dot{\bar{q}}+K_{a} \bar{p}+L_{a} \dot{\bar{v}}=F_{l} \bar{v} \\
\bar{q}-\dot{\bar{p}}=0
\end{array}\right\} \text { for breathing flow }
\end{aligned}
$$

where $\bar{v}$ and $\bar{u}$ are nodal velocity and displacement vectors, $\bar{p}$ the nodal pressure vector, $\mathrm{M}, \mathrm{C}, \mathrm{K}$, and $\mathrm{G}$ are the mass matrix, the damping matrix, stiffness matrix, and the coefficient matrix of the pulsating pressure, $\mathrm{Ma}$, and $\mathrm{Ka}$ 
the mass matrix and stiffness matrix of flow, and La and Fl the motion vector coefficients matrices of the vocal chord respectively.

The complex eigen frequency of the governing equations can be solved using equation (33) by the root locus approach. When the state variables of the vocal chord are assumed to be expressed by

$$
\bar{v}=V e^{\lambda t}, \quad \bar{u}=U e^{\lambda t}, \quad \bar{q}=Q e^{\lambda t}, \quad \bar{p}=P e^{\lambda t}
$$

where $\lambda\left(=\lambda_{R}+j \lambda_{i}\right)$ is the complex eigen value, then substituting equations (34) into equations (33), we obtain

$$
\left[\begin{array}{cccc}
M & 0 & 0 & 0 \\
0 & -I & 0 & 0 \\
L a & 0 & M a & 0 \\
0 & 0 & 0 & -I
\end{array}\right] \lambda\left\{\begin{array}{l}
V \\
U \\
Q \\
P
\end{array}\right\}=-\left[\begin{array}{cccc}
C & K & 0 & G \\
I & 0 & 0 & 0 \\
-F l & 0 & 0 & K a \\
0 & 0 & I & 0
\end{array}\right]\left\{\begin{array}{l}
V \\
U \\
Q \\
P
\end{array}\right\}
$$

Once the complex eigen frequencies are obtained from equation (35), the vocalizing condition can be determined as the intersection of both the damped vibration region with $\lambda_{R}<0$ and the divergent vibration region with $\lambda_{R}>0$ as shown in Figure 6.

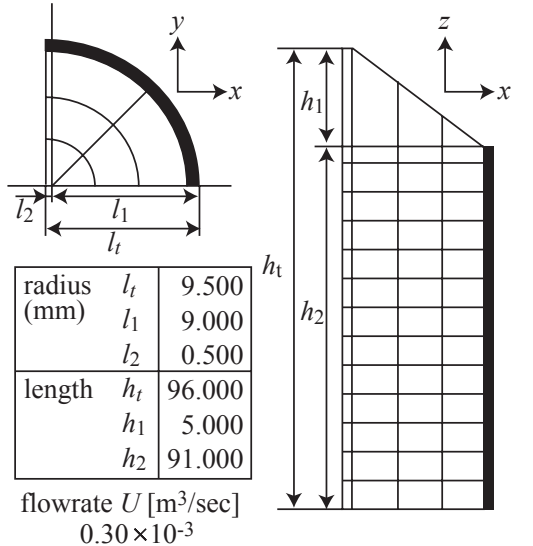

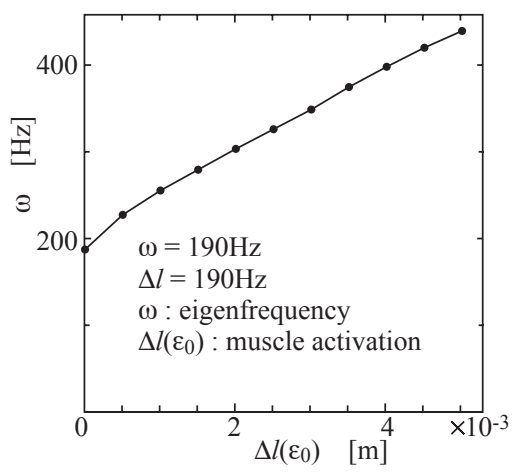

Figure 5: Calculation model of Figure 6: Engenfrequency characteristics breathing flow in trachea. under muscle activation.

\section{Applications to the actual system and experiments}

\subsection{The calculation models}

In order to examine the applicability of the results obtained from above approaches, a pharynx vocal chord system of a living human (male), 22 years old, is measured by MRI equipment, and solved using the models shown in Figure 3 for the vocal chord and in Figure 5 for the trachea respectively. The 
vocal chord is spritted into left and right vocal folds along the sagittal plane as shown in Figure 1(b), and when breathing, the two muscles which are stored in each vocal fold, and fixed at one longitudinal end to the thyroid cartilage, and another fixed at another end to cricoid cartilage, is open state as shown in Figure 1(c), and when vocalizing, two vocal folds are closed in each other, the oscillation cycle due to the pressure rise of the breathing flow begin to starts.

Along with the actual muscles construction shown in Figure 1(d), the calculation model, shown in Figure 3, has two muscle layers and one physiological damping(water) layer, stored in mid layer zone. The principal dimensions and physical nature of the materials are given in the table of Figure 3, and Tables 1 and 2.

Table 1: Constitutive parameters of vocal soft tissue $\left(\times 10^{4} \mathrm{~Pa}\right)$.

\begin{tabular}{|c|c|c|c|c|}
\hline$i$ & $\mathrm{a}$ & $\mathrm{b}$ & $\mathrm{c}$ & $\mathrm{d}$ \\
\hline$a_{i}$ & 3.66 & 2.10 & 0.054 & 4.85 \\
\hline$\mu_{i}$ & 22.1 & 16.11 & 36.66 & 1.25 \\
\hline$c_{i}$ & 0.0469 & 0.0954 & 0.0210 & 0.0615 \\
\hline
\end{tabular}

Table 2: $\quad$ Muscle parameters.

constitutive

\begin{tabular}{|c|c|c|c|c|c|c|}
\hline Act. & $c_{1}$ & $c_{2}$ & $c_{3}$ & $\mu_{1}$ & $\mu_{2}$ & $S_{\max }^{0}$ \\
\hline & 1.04 & 5.82 & 0.894 & 0.689 & 1.45 & 1.45 \\
\hline Pas. & $\psi_{1}$ & $\psi_{2}$ & $\psi_{3}$ & $\psi_{4}$ & $C_{m}$ & $C_{m}$ \\
\hline & 87.0 & 3.30 & 1.88 & 5.78 & 8.08 & 8.08 \\
\hline
\end{tabular}

At the interface between the behind plane of the vocal muscle and the breathing flow, the vocal chord is subjected to the pulsating pressure of the breathing flow due to the open-closed cycle of vocal hold, and the breathing flow is subjected to the pulsating acceleration due to the vocal chord vibration as shown in Figure 4.

The calculation model of the breathing flow and the principal dimension of the model are shown in Figure 5.

\subsection{Calculation results of the vocal chord}

The eigen frequencies of the vocal chord has been analysed at first, by varying the activation level of vocal muscle shown in Figure 1(d) as A.

The result is shown in Figure 6, and it is seen that the eigenfrequency of the vocal chord is $190 \mathrm{~Hz}$ without any muscle activation, and it increases due to the muscle activation level, $\Delta l\left(\varepsilon_{o}\right)$, defined in Figure 2(e), in which the dashed stress(or force) is induced by partial pull back, $\Delta l\left(\beta_{2}\right)$, of the isotropic contraction $l_{0}\left(\beta_{2}\right)$.

Figure 7 shows the results of complex eigenvalue analysis of the vocal chord without muscle activation.

It is seen in Figure 7 that the damped vibration occurs in the negative region of the real root as shown in Figure 7(a), and the divergent vibration in the positive region of the real root is occurred as shown in Figure 7(c).

On the contrary, the steady, constant amplitude vibration is successfully occurred at the intersection between the damped and the divergent vibrations, and it give rise to the source voice vocalizing. 
The eigenfrequencies of (a) through (c) cases coincides each other with the value of $190 \mathrm{~Hz}$ which is the same as in Figure 6.

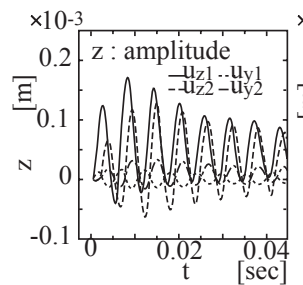

(a) damped vibration

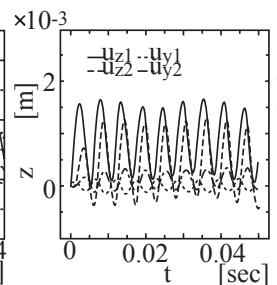

(b) steady vibration

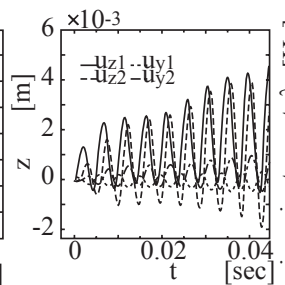

(c) divergent vibration

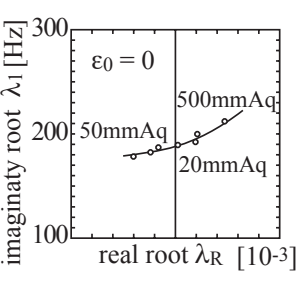

(d) result of route-locus approach

Figure 7: Damped, divergent and steady vibrations characteristics by route locus approach $\left(\varepsilon_{0}=0\right)$.

\subsection{Coupled analysis of the vocal chord vibration and breathing flow dynamics}

Finally the coupled analysis of both the vocal chord vibration and the breathing flow dynamics has been carried out, and the result is shown in Figure 8(a) to (c), in which Figure 8(a) show the time history of the vibration amplitude at the starting and Figure 8(b) shows the cooperation characteristics of the pressure pulsation in flow dynamics and vibration amplitude.

During the vocal-hold opening, the pressure in pulsating flow reduces, and the vibration amplitude reaches to the maximum value.

Once the vocal-hold closes due to the reduction of vibration amplitude, the pressure in the breathing flow increase to the maximum value, yielding to the vibration amplitude increases.

When this cycle of iteration continue in steady state, and that it coincides with the eigenfrequency of the vocal chord, it results to the source voice vocalizing, which can be applied to generates vowels or consonants at the connected resonant box of the mouth cavity-tongue motion.

Figure $8(\mathrm{c})$ shows the results of the route locus approach both for the case without vocal muscle activation and for the case with the vocal muscle activation with $\varepsilon_{0}=0.3$, which is estimated to generate the one-octave higher frequency of the source voice. It is successfully appeared in the calculation results.

\subsection{Measurements of the source voice vocalizing}

Two types of measurements for the source voice vocalizing of a 22-year old living human have been done, i.e.

One is vibration measurements at the Laryngeal Prominence, using the acceleration pick up and another is the measurements of vocalizing flow at the mouth-outlet part using microphone, followed by FFT analyses.

Each measurement has two cases, that is, one is for vocalizing the standard [a] and another for vocalizing of [a'] which has one octave higher than that of standard. 


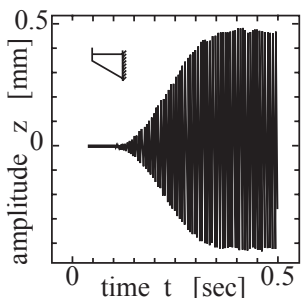

(a) amplitude-time curve in steady vibration $\left(\varepsilon_{0}=0\right)$

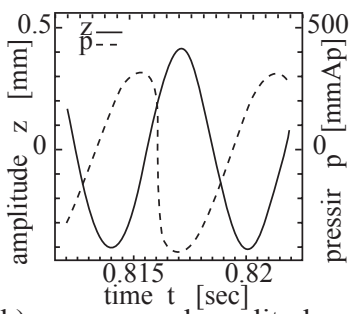

(b) pressure and amplitude curves in breathing flow $\left(\varepsilon_{0}=0\right)$

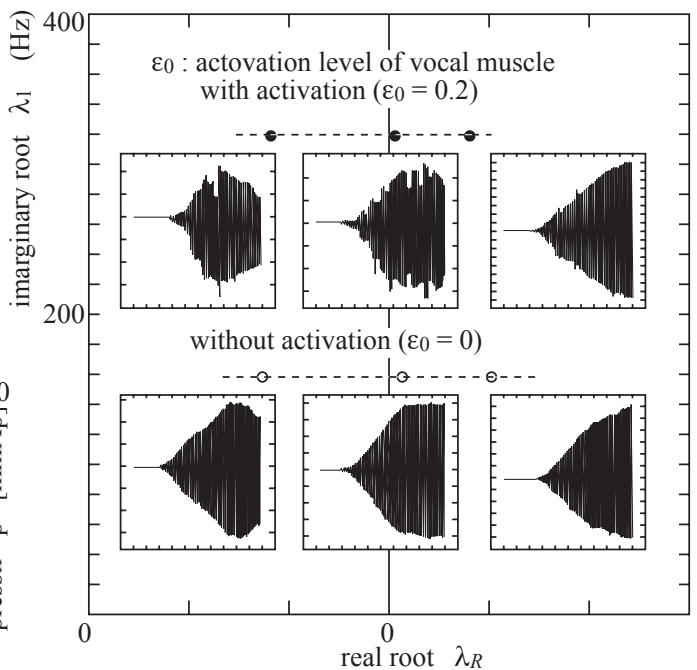

(c) results of root locus approach

Figure 8: Coupled calculation results of the vocal chord vibration and breathing flow dynamics.

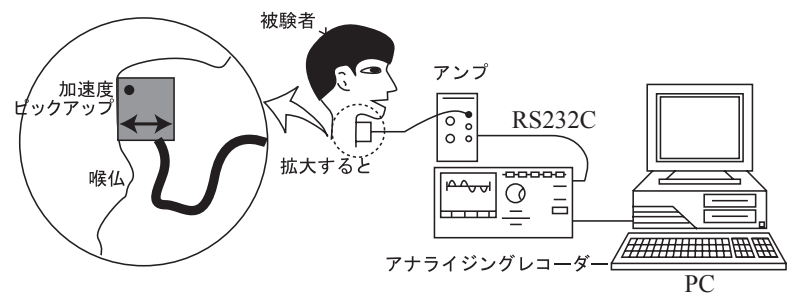

(a) measurement equipments for vibration of the laryngeal prominence

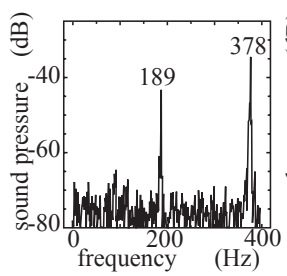

(b-1) frequency resuponse (b-2) frequency resuponse characteristics for $[\mathrm{a}]$ characteristics for $\left[\mathrm{a}^{\prime}\right]$ with withoutmuscle acrivation muscle acrivation, $\varepsilon_{0}=0.3$

(b) vibration measurement results

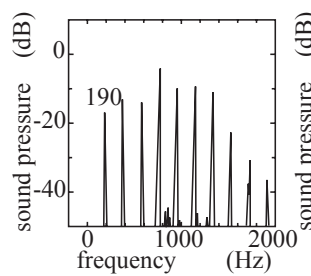

(c-1) frequency resuponse characteristics for $\left[a^{\prime}\right]$ with withoutmuscle acrivation muscle acrivation, $\varepsilon_{0}=0.3$

(c) results of sound pressure measurements

Figure 9: Measurement results of a living human vocalizing [a][a'] for source voice. 
Both results are shown in Figure 9(a) through (c).

Figure 9(a) shows the measurement equipments for the vibration at the Laryngeal Prominence. Figure 9(b-1) shows the results of the frequencyresponse characteristics of the vibration for vocalizing [a] without muscle activation, and Figure 9(b-2) shows the similar results for vocalizing [a'] with muscle activation of $\varepsilon_{0}=0.3$, respectively.

Figure 9(c-1) shows the results of the frequency-response characteristics of the sound pressure measurements for [a] and Figure 9(c-2) for [a'] respectively.

It is seen from these figures as follows.

(1) The results, obtained from the vibration measurements, and from the sound pressure measurements, coincide well in each other.

(2) The exciting frequency of standard source voice [a] coincides well with those of the calculated as shown in Figure 6 and Figure 8(c) without muscle activation.

(3) The exciting frequency of one-octave higher than standard [a'] coincides well with those of the calculated as shown in the same figures.

(4) By comparisons with those obtained from both the calculated and the measured for one-octave higher exciting frequency. It is clarified that the exciting frequency for [a'] vocalization can be obtained from the vocal muscle activation of the pull back contents of $20 \%$ of the muscle length.

\section{Concluding remarks}

The results are summarized as follows.

(1) The three-dimensional finite element system for the dynamic response analysis of muscle and hyperelastic finite deformation soft tissue has been developed.

(2) Combined it with the finite element system of flow dynamics of the Lighthill equation, the coupled dynamics of the vocal chord vibration and breathing flow dynamics has been successfully solved by varying the vocal muscle activation.

(3) Complex eigenfrequency analysis of the vocal chord has successfully revealed the characteristics of the presence of the damped vibration, the divergent vibration and the steady amplitude vibration, and it is verified that the steady amplitude vibration can create the source voice vocalizing.

(4) It is also demonstrated that the various frequencies of the source voice vocalizing can be obtained by varying the vocal muscle activation.

(5) The exciting frequency, measured at Laryngeal Prominence, for the source voice vibration, and the exciting frequency of the acoustical vibration, measured at mouth outlet part are coincides well in each other, and that they coincides well with those obtained from calculated results.

(6) It is also verified that the exciting frequency of the one octave higher source voice vocalizing [a'], which is obtained from measurements, can be attained by vocal muscle activation of $20 \%$ pull back of the free activation contraction, based on the numerical calculation results. 


\section{References}

[1] Ishizaka, J. Flanagan (1978). Self exciting vibration model in source voice vocalizing of vocal chord, J. of the Acoustical Society of Japan.

[2] Kagawa (1989). Sound and Vibration Engineering using the Finite Element Method, Baifukan.

[3] Tsuta, T., Wang, C., and Yamane, K. (1992). Compute Simulation of Facial Mechanics(1st) Finite Element Method of Hyper elastic Finite Deformation Solid, Trans, JSME.

[4] Tsuta, T., Li, M., Iwamoto, T., and Kaneko, O. (1997). Muscle Continuum Mechanics and FEM Simulation on Facial Mechanics of Muscle-Soft Tissue System. Engineering Research Report of Hiroshima Univ. Vol.46.

[5] Tsuta, T., and Iwamoto, T. (2001). Vowel Vocalizing Analyses of Mouth Lavity-Tongue Muscle System Using Frequency Optimization Approach of Source Voice Pulsating Flow, Proc. APCOM'01.

[6] Ishizaka, Matsudaira (1972). Fluid Mechanical considerations of Vocal Chord Vibration, SCRL Monograph 8.

[7] Iijima, Miki, N and Nagai, N. (1991). Mechanical Influences of Nonlinear Viscoelastic Flow in Vocal Chord Surface, IEICE J75-A11.

[8] Tsuta, T., Iwamoto, T. and Fujimura, T. (1999). Continuum Mechanics of Facial Muscles and Creep-Relaxation Remodeling Analysis of Muscle-Skin System. CMBBE. Vol.4. 